\title{
JORDAN TRIPLE SYSTEMS, R-SPACES, AND BOUNDED SYMMETRIC DOMAINS
}

\author{
BY OTTMAR LOOS
}

Communicated by N. Jacobson, January 6, 1971

Abstract. In this note, we establish a one-to-one correspondence between compact Jordan triple systems (see below for the definition) and symmetric $R$-spaces (i.e., symmetric spaces which are quotients of semisimple Lie groups by parabolic subgroups, see [7]). We obtain a simple geometric characterization of symmetric $R$-spaces among compact symmetric spaces. The noncompact dual of a symmetric $R$-space may be realized as a bounded domain $D$ in a real vector space. There is a one-to-one correspondence between boundary components of $D$ and idempotents of the corresponding Jordan triple system. Using this, we generalize the results of WolfKoranyi [8] to the real case. In particular, the image of $D$ under a generalized Cayley transformation is the real equivalent of a Siegel domain of type III.

1. Jordan triple systems. A Jordan triple system (=JTS) (see [2], [3]) is a vector space $V$ together with a trilinear map $V \times V \times V$ $\rightarrow V,(x, y, z) \mapsto\{x y z\}$, satisfying the following identities:

$$
\begin{gathered}
\{x y z\}=\{z y x\}, \\
\{u v\{x y z\}\}=\{\{u v x\} y z\}-\{x\{v u y\} z\}+\{x y\{u v z\}\} .
\end{gathered}
$$

For $x, y \in V$ we define the linear map $L(x, y)$ of $V$ by $L(x, y)(z)$ $=\{x y z\}$. A finite-dimensional real JTS is called compact if the quadratic form $x \mapsto \operatorname{trace} L(x, x)$ is positive definite. From now on, $V$ will denote a compact JTS. Then $V$ becomes a Euclidean vector space with the scalar product $(x, y)=\operatorname{trace} L(x, y)$. By (2), the vector space $\mathfrak{S}$ spanned by $\{L(x, y): x, y \in V\}$ is a Lie algebra of linear transformations of $V$, closed under taking transposes with respect to $\left(\right.$, ). Thus the contragredient $\mathfrak{S}$-module $V^{\prime}$ of $V$ may be identified with $V$ as a vector space, and $X \cdot v^{\prime}=-{ }^{t} X\left(v^{\prime}\right)$, for $X \in \mathfrak{S}$ and $v^{\prime} \in V^{\prime}$.

TheOREM 1 (Koecher). (a) $\mathfrak{R}=V+\mathfrak{S}+V^{\prime}$ becomes a semisimple Lie algebra with the definitions

$$
[X, Y]=X Y-Y X, \quad[X, v]=-[v, X]=X \cdot v,
$$

AMS 1970 subject classifications. Primary 17B60, 17E05, 32M15, 53C35.

Key words and phrases. Jordan triple system, symmetric $R$-space, bounded symmetric domain, boundary component, idempotent, Cayley transformation.

1 Research supported by Deutsche Forschungsgemeinschaft. 
for $X, Y$ in $\mathfrak{S}$ and $v \in V \cup V^{\prime}$;

$$
[V, V]=\left[V^{\prime}, V^{\prime}\right]=0, \quad\left[u, v^{\prime}\right]=-2 L(u, v),
$$

for $u \in V$ and $v^{\prime} \in V^{\prime}$.

(b) $Z=-\operatorname{Id}_{V}$ belongs to $\mathfrak{S},(\operatorname{ad} Z)^{3}=$ ad $Z$, and the $-1-, 0-,+1-$ eigenspaces of ad $Z$ are $V, \mathfrak{S}, V^{\prime}$.

It is easily seen that the map $\tau: X \mapsto-{ }^{t} X(X \in \mathfrak{S}), v \mapsto v^{\prime}, v^{\prime} \mapsto v$, is a Cartan involution of $\mathfrak{R}$, and that $\sigma|\mathfrak{S}=+1, \sigma| V+V^{\prime}=-1$ defines an involutive automorphism $\sigma$ of $\&$ commuting with $\tau$.

2. Symmetric $R$-spaces. Keeping the above notations, let $L$ be the centerfree connected Lie group with Lie algebra $\&$, let $H$ be the centralizer of $Z$ in $L$, let $U$ be the maximal compact subgroup of $L$ determined by $\tau$, and let $K=U \cap H$. Then $K$ lies between the full fixed point set of $\sigma$ in $U$ and its identity component. The normalizer $P$ of $V$ in $L$ is parabolic, and we have $U / K \cong L / P$. Thus $M=U / K$ is a symmetric $R$-space (cf. [7]).

TheOREM 2. The map $V \mapsto M$ establishes a one-to-one correspondence between isomorphism classes of compact JTS and symmetric R-spaces.

Let $M$ be a compact symmetric space, $o$ a point of $M$, and $A$ a maximal torus of $M$ containing $o$. The tangent space of $A$ at $o$ is denoted by $T_{o}(A)$. Then $\Lambda(M)=\left\{v \in T_{o}(A): \operatorname{Exp} v=0\right\}$ is a lattice in $T_{o}(A)$, the unit lattice of $M$ (with respect to $A$ ). We say that $M$ has cubic unit lattice if there exists a Riemannian metric on $M$, invariant under all symmetries, and an orthonormal basis $e_{1}, \cdots, e_{r}$ of $T_{o}(A)$ with respect to this metric, such that $\Lambda(M)=\sum \boldsymbol{Z} \cdot e_{i}$.

Theorem 3. A compact symmetric space is a symmetric $R$-space if and only if it has cubic unit lattice.

As a special case, we obtain the following characterization of the classical groups: A compact connected Lie group is a direct product of the groups $\mathrm{SO}(n), \mathrm{U}(n), \mathrm{Sp}(n)$ if and only if it has cubic unit lattice. The fact that symmetric $R$-spaces have cubic unit lattice is contained in [7]. Theorem 3 may be used to give a global classification of symmetric $R$-spaces (and hence of compact JTS). The classification has been obtained by different methods in [1] and [4].

3. Bounded symmetric domains. Keeping the previous notations, let $G$ be the connected fixed point set of $\sigma \tau$ in $L$. Then $M^{*}=G / K_{0}$ is the noncompact dual of $M$. There is an imbedding $\zeta: M^{*} \rightarrow V$ such that $g \equiv \exp \left(\zeta\left(g K_{0}\right)\right) \bmod P$, for $g \in G$. The image $D=\zeta\left(M^{*}\right)$ is a 
bounded domain in $V$ (see [4], [7]) which inherits a Riemannian metric from $M^{*}$. This generalizes the Harish-Chandra imbedding of a Hermitian symmetric space of noncompact type. Following Pjateckii-Shapiro [5], two points $x, y$ in $\bar{D}$ (the closure of $D$ in $V$ ) will be called equivalent if there exist sequences $\left(x_{n}\right),\left(y_{n}\right)$ in $D$, converging to $x, y$ respectively, such that the Riemannian distance of $x_{n}$ and $y_{n}$ remains bounded. The equivalence classes are the metric boundary components of $D$. We also define affine boundary components as follows. By a segment in $V$ we mean a set of the form $\{a+t b: 0<t<1\}$ where $a, b \in V$. Then a subset $F$ of $\bar{D}$ is called an affine boundary component if (1) every segment contained in $\bar{D}$ and meeting $F$ is contained in $F,(2)$ no proper nonempty subset of $F$ satisfies (1).

An element $c$ of $V$ is called an idempotent if $\{c c c\}=c$. Part of the following theorem is due to $\mathrm{K}$. Meyberg (unpublished).

Theorem 4. (a) There is a Peirce-decomposition $V=V_{1}(c)+V_{1 / 2}(c)$ $+V_{0}(c)$ where $V_{i}(c)$ is the eigenspace of $L(c, c)$ corresponding to the eigenvalue $i$.

(b) With $x \circ y=\{x c y\}, V_{1}(c)$ is a real semisimple Jordan algebra with unit element $c$. The map $x \mapsto \bar{x}=\{c x c\}$ is a Cartan involution of $V_{1}(c)$; in particular, $V_{1}^{+}(c)=\left\{x \in V_{1}(c): \bar{x}=x\right\}$ is a formally real Jordan algebra.

(c) With the induced multiplication, $V_{0}(c)$ is a compact JTS.

Now we can describe the relation between boundary components and idempotents.

THEOREM 5. Metric and affine boundary components coincide; they are precisely the sets $F_{c}=c+\left(D \cap V_{0}(c)\right)$ where $c$ is an idempotent. $D_{c}=D \cap V_{0}(c)$ is the bounded symmetric domain belonging to the compact JTS $V_{0}(c)$.

This allows us to recover all the results of [8]. In particular, the space of boundary components of a given type is a fibre bundle over a compact symmetric space, and the stability group of a boundary component in $G$ is parabolic. Finally, we define the Cayley transformation belonging to an idempotent $c$ (resp. a boundary component $F_{c}$ ) to be $\gamma_{c}=\exp \frac{1}{4} \pi(c+\tau(c))$. For $z \in D_{c}$ and $x, y \in V_{1 / 2}(c)$ set $\Phi_{z}(x, y)=\left\{x,(\operatorname{Id}+\mu(z))^{-1}(y), c\right\}$ where $\mu(z)(y)=2\{c y z\}$. Also for $u \in V_{1}(c)$ let $\operatorname{Re} u=\frac{1}{2}(u+\bar{u}) \in V_{1}^{+}(c)$, and denote by $Y$ the interior of the set of squares of $V_{1}^{+}(c)$. This is the self-dual cone (domain of positivity) associated with the formally real Jordan algebra $V_{1}^{+}(c)$. 
Theorem 6. The image of $D$ under the Cayley transformation $\gamma_{c}$ is the set of all $x+y+z \in V_{1}(c)+V_{1 / 2}(c)+V_{0}(c)$ such that $z \in D_{c}$ and $\operatorname{Re}\left(x-\Phi_{z}(y, y)\right) \in Y$, a real Siegel domain of type III.

In the special case where $c$ is a maximal idempotent, i.e., $V_{0}(c)=0$, we have $\gamma_{c}(D)=\left\{x+y \in V_{1}(c)+V_{1 / 2}(c): \operatorname{Re} x-\{y y c\} \in Y\right\}$, a real Siegel domain of type II. This result is due to Takeuchi [7], see also [6].

\section{REFERENCES}

1. S. Kobayashi and T. Nagano, On filtered Lie algebras and geometric structures. I, J. Math. Mech. 13 (1964), 875-907. MR 29 \#5961.

2. M. Koecher, An elementary approach to bounded symmetric domains, Lecture Notes, Rice University, Houston, Texas, 1969.

3. K. Meyberg, Jordan-Tripelsysteme und die Koecher-Konstruktion von LieAlgebren, Math. Z. 115 (1970), 58-78.

4. T. Nagano, Transformation groups on compact symmetric spaces, Trans. Amer. Math. Soc. 118 (1965), 428-453. MR 32 \#419.

5. I. I. Pjatecki1-Šapiro, Geometry of classical domains and theory of automorphic functions, Fizmatgiz, Moscow, 1961; French transl., Dunod, Paris, 1966; English transl., Gordon and Breach, New York, 1967. MR 25 \#231; MR 33 \#5949.

6. V. I. Semjanistiú, Symmetric domains and Jordan algebras, Dokl. Akad. Nauk SSSR 190 (1970), 788-791 = Soviet Math. Dokl. 11 (1970), 215-218.

7. M. Takeuchi, Cell decompositions and Morse equalities on certain symmetric spaces, J. Fac. Sci. Univ. Tokyo Sect. I 12 (1965), 81-192. MR 35 \#7350.

8. J. A. Wolf and A. Koranyi, Generalized Cayley transformations of bounded symmetric domains, Amer. J. Math. 87 (1965), 899-939. MR 33 \#229.

Mathematisches Institut Der UniversitäT, 8 MÜNChen 13, SChellingstrasse 2-8, Germany 